\title{
TWO-TEMPERATURE TRANSONIC ACCRETION DISKS
}

\author{
K.E.NAKAMURA ${ }^{1}$, M.KUSUNOSE $^{2}$, R.MATSUMOTO $^{3}$, S.KATO $^{1}$ \\ ${ }^{1}$ Dept. of Astronomy, Kyoto Univ., Kyoto 606-01 \\ ${ }^{2}$ Dept. of Physics, Kwansei Gakuin Univ., Nishinomiya 662 \\ ${ }^{3}$ Dept. of Physics, Chiba Univ., Chiba 263
}

\section{Introduction}

The optically thin, advection-dominated accretion flows are thermally stable against global perturbations. In addition, they have high temperatures because of inefficient radiative cooling. They are thus promising candidates of models to explain the high energy emission of X-ray stars and AGNs. So far, models, however, take no account of the advective heat transport in determining the thermal structure of the electron system. The validly of this neglect, however, must be checked by integrating the electron energy equation globally as well as the ion energy one.

\section{Results}

In the inner region of the disks, we find that in the ion gas the viscous heating is roughly balanced by advective cooling. That is, the viscously heated ions are transported inward without being cooled. The approximation of advection-dominated flows is good for the ion gas, and only a small fraction of energy is transported to the electron gas by the Coulomb collisions $\left(\Lambda_{\mathrm{ie}}\right)$. When the energy balance in the electron gas or the spectrum from the disk are concerned, the following approximation are sometimes adopted: after the ion temperature is calculated by the approximation of advectiondominated flow, the electron temperature is determined under the approximation of $\Lambda_{\mathrm{ie}}=Q_{\mathrm{rad}}^{-}$. Our results, however, show that the compressive heating of the electron gas is the main ingredient to keep the electron temperature against strong synchrotron cooling of the electron gas. We should take the approximation of $Q_{\mathrm{adv}, \mathrm{e}}+Q_{\mathrm{rad}}^{-} \sim 0$ with a negative $Q_{\mathrm{adv}}$ (advective heating), instead of $\Lambda_{\mathrm{ie}}=Q_{\mathrm{rad}}^{-}$. 